\title{
Typing safe antibiotics in amoxicillin hypersensitive patients — development of a stepwise protocol
}

The authors declare no financial disclosure.

\section{Abstract}

Introduction: A history of an adverse reaction to amoxicillin, irrespective of the mechanism involved, significantly elevates patients' anxiety and affects therapeutic decisions in the future, leading to unnecessary avoidance of antibiotics. As a consequence, it would be useful to find a safe and reliable protocol for typing safe alternative antibiotics.

The aim of the study was to determine negative predictive value of typing safe antibiotic in patients with a history of hypersensitivity reaction to amoxicillin.

Material and methods: 71 patients, aged 20-83, with a history of an adverse reaction to amoxicillin were retrospectively analysed. $\mathrm{On}$ the basis of the reaction type they were divided into three groups: A - symptoms not typical for hypersensitivity reactions, $B$ - allergy manifested by urticaria and/or angioedema, $C$ - anaphylaxis. In group A amoxicillin was tested, in group $\mathrm{B}$ - cefuroxime, and in group $\mathrm{C}$ - macrolide: azithromycin or clarithromycin. Telephone follow-up visits were performed twice: $6-12$ months and $3-5$ years after the clinical assessment to evaluate tolerance of antibiotics. On the basis of the follow-up results, the negative predictive value (NPV) of the protocol was calculated.

Results: The full diagnostic protocol was applied in 62 participants. Amoxicillin was found safe in 22, cefuroxime - in 21 and macrolide - in 19 patients. No anaphylactic reactions were observed during the tests. On the basis of the telephone follow-up, the NPV of the protocol was $96 \%$ in the first follow-up and $97 \%$ in the second one.

Conclusion: A stepwise approach including SPTs, ICTs and provocations with amoxicillin / cefuroxime/macrolide — depending on a patient's history — is safe and allows typing an antibiotic in the vast majority of patients.

Key words: beta-lactam allergy, drug allergy management, drug challenge test, skin tests, drug typing

Pneumonol Alergol Pol 2016; 84: 16-21

\section{Introduction}

Beta-lactams are widely used antibiotics, particularly in the therapy of respiratory and urinary tract infections. According to both Polish and international guidelines, amoxicillin, either alone or combined with clavulate, is the first-choice antibiotic in therapy of pneumonia, sinusitis, otitis media and other common bacterial infections [1, 2]. Although beta-lactams are generally considered safe, patients often report adverse reactions to them $[3,4]$.
Adverse drug reactions can be classified into two major types: A - grouping those dose-dependent and predictable, and B - not dose-dependent and unpredictable. In relation to beta-lactams, type A reactions include, e.g. diarrhoea, pseudomembranous colitis or candidosis resulting from a change in the bacterial flora. On the contrary, allergy is a type $\mathrm{B}$, immune reaction observed only in a group of sensitized individuals.

A history of an adverse reaction to an antibiotic, irrespective of a mechanism involved, significantly elevates patients' anxiety and affects 


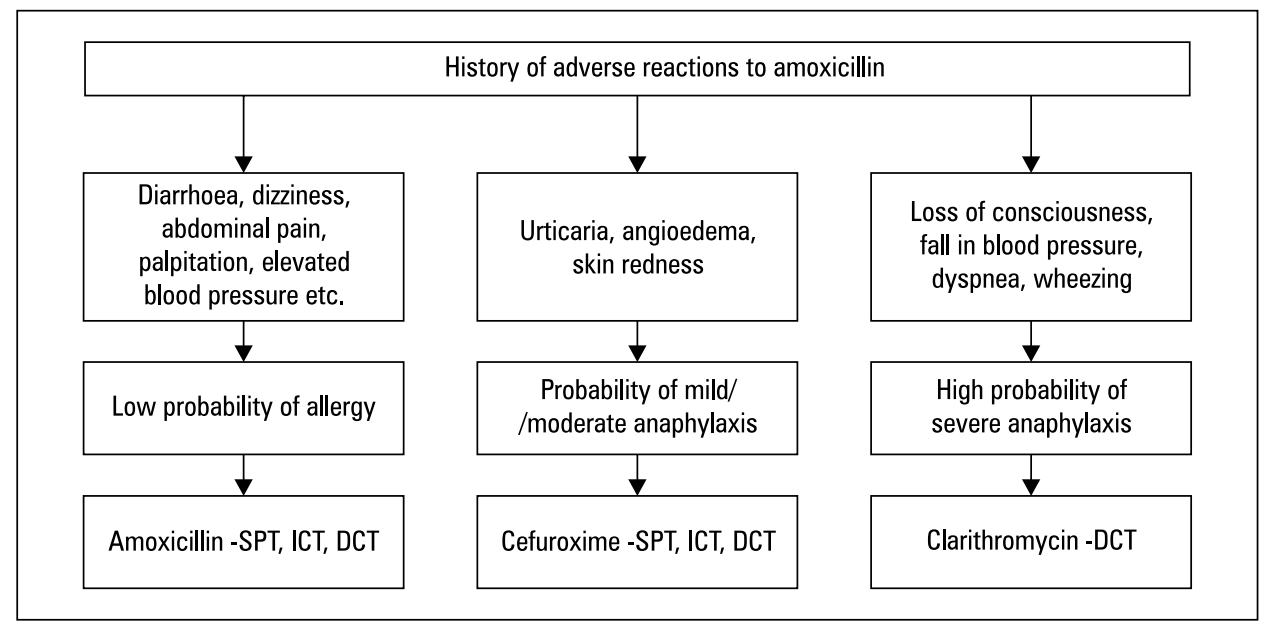

SPT — skin prick test; ICT — intracutaneous test; DCT — drug challenge test

Figure 1. Algorithm of choosing antibiotic for typing

therapeutic decisions in the future. In some cases, the whole group of beta-lactams is advised to be avoided or a decision to start therapy is delayed. As a consequence, it seems necessary to find a safe and reliable protocol for typing safe antibiotics for patients with history of hypersensitivity reaction related to amoxicillin. In the literature several such protocols have been described which aim at confirming hypersensitivity [5, 6]. Most of them were based on skin tests and drug provocation tests (DPT). However, little is known about their negative predictive value in 'real life' conditions.

The aim of the study was to determine negative predictive value for typing safe antibiotics in patients with a history of allergy to amoxicillin.

\section{Material and methods}

71 patients, including 68 women and 3 men, with a history of an adverse reaction to amoxicillin were retrospectively analysed. The mean age at the time of drug typing was 53 years (range 20-82 years). The participants were hospitalized at the Department of Allergology, Medical University of Gdańsk from January 2010 to December 2012. Exclusion criteria included: pregnancy, acute infection, beta-blockers intake, unstable circulatory insufficiency and any other situation that could, in our opinion, affect the patients' safety or reliability of the tests. Before inclusion, the patients gave written informed consent for all the procedures.

On the basis of the history of the reaction to amoxicillin patients were divided into three groups: A - symptoms not typical for hypersensitivity reactions (diarrhoea, heart palpitations, malaise, etc.), B - allergy manifested by urticaria and/or angioedema, C - anaphylaxis - as defined by Sampson et al. [7] (Fig. 1). Our diagnostic approach varied between the groups. In group A, amoxicillin was tested as the probability of true allergy was considered low. In patients from group B, allergy was assessed to be probable, but there was no history of anaphylaxis. Thus, they were tested with cefuroxime. Finally, group C, with a history of anaphylaxis related to amoxicillin, was provoked with macrolide (azithromycin or clarithromycin).

Before drug typing medications that could affect the results of the tests were withdrawn according to general guidelines [8]. A stepwise typing of a safe beta-lactam (amoxicillin, cefuroxime) included skin prick tests (SPT), intracutaneous tests (ICT) and oral drug provocation tests (DPT). The positive control was a prick test with histamine and negative - with saline. In SPTs and ICTs, the drugs' concentrations applied were 20 $\mathrm{mg} / \mathrm{ml}$ for amoxicillin and $2 \mathrm{mg} / \mathrm{ml}$ for cefuroxime. Skin prick tests were considered positive if a wheal of $3 \mathrm{~mm}$ or more in diameter was found. Intracutaneous tests were assessed as positive in case of a wheal of $5 \mathrm{~mm}$ in diameter or more with erythema. Skin tests were followed by oral, single-blind, placebo-controlled drug provocation tests. Doses were administered every 60 minutes $(10,90,150,250,500 \mathrm{mg}$ of amoxicillin or 5,95 , $150,250 \mathrm{mg}$ of cefuroxime).

Typing of a macrolide (clarithromycin or azithromycin) included single-blind, placebo-controlled oral provocation tests with increasing doses of the drug: 5, 95, 150, $250 \mathrm{mg}$ (every 60 minutes).

In all the protocols, if one of the steps yielded a reaction, the drug was excluded from fur- 
Table 1. Results of skin prick tests (SPT), intracutaneous tests (ICT) and drug provocation tests (DPT) with amoxicillin, cefuroxime and macrolides

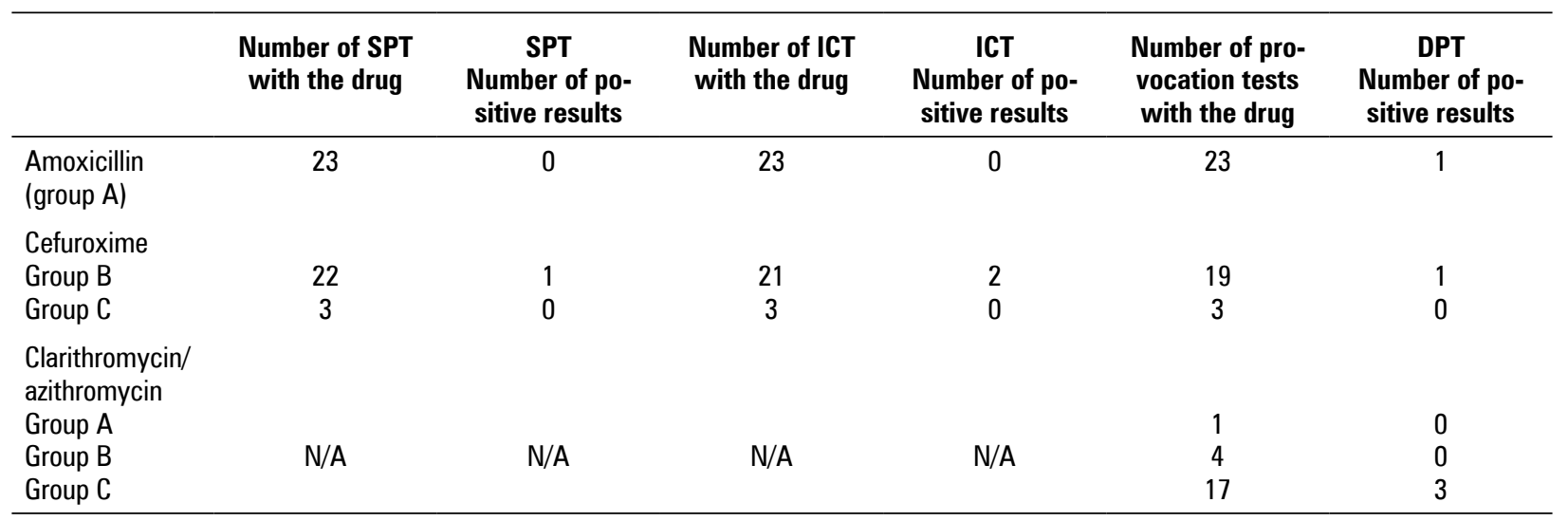

ther tests. In cases of hypersensitivity reaction, provocation was ceased and, after 24 hours free from symptoms, another antibiotic was tested (macrolide - in cases of reaction to beta-lactam and vice versa).

Telephone follow-up visits were performed 6-12 months and 3-5 years after drug typing. Patients were asked questions about antibiotics used since the visit in the clinic and adverse reactions related to their administration. On the basis of the follow-up results, negative predictive value (NPV) of the typing protocol was calculated.

\section{Results}

71 patients were admitted to the Department of Allergology for typing of a safe antibiotic. 39 of them had a history of immediate hypersensitivity reaction after administration of amoxicillin and 32 - amoxicillin with clavulate. Patients reported: urticaria - 34 cases, angioedema 24 , skin redness -20 , shortness of breath -19 , abdominal pain -16 , nausea -14 , diarrhoea -8 , vomiting -4 , loss of consciousness -8 , dizziness -6 , malaise -26 . As a consequence, 28 patients were assigned to group A, $25-$ group B and 18 - group C. 15 patients reported reactions to other antibiotics as well, mostly to macrolides (9 patients) followed by clindamycin ( 2 patients), doxycycline ( 2 patients), sulfamethoxazole+trimethoprim ( 2 patients) and ciprofloxacin (1 patient).

During the tests one patient withdrew informed consent and eight reacted to placebo. Thus, only in 62 participants (group A $-23, \mathrm{~B}-22$, $C-17$ ) full protocol was performed and this group was included into analysis.
The results of skin tests and provocations with antibiotics are presented in Table 1. In group A, most of the participants had negative skin tests and provocation tests with amoxicillin. One patient developed urticaria during provocation with amoxicillin and a macrolide was found safe for him. In group B, 18 participants tolerated cefuroxime, in 3 cases positive results of skin test were found and in 1 case - provocation test was positive. In all the positive responders macrolide was found safe. In group C, 14 patients have not had any symptoms of hypersensitivity to macrolide. In 3 positive responders cefuroxime was found safe.

Finally, in all participants who did not respond to placebo it was possible to type a safe antibiotic. Amoxicillin was found safe in 22 cases, cefuroxime - in 21 and macrolide - in 19 patients.

The results of a telephone follow-up are presented in Table 2. After 6-12 months it was possible to contact $50(80 \%)$ participants. A drug typed at a clinical visit was administered in 28 patients and 25 reported no reactions. Two patients reported diarrhoea and abdominal pain they experienced after 4-5 days of the therapy with amoxicillin. The symptoms resolved spontaneously and were not accompanied by any typical symptom of hypersensitivity reaction. One patient had delayed skin reaction (papular rash with itching) after 3-day therapy with azithromycin. As we did not consider isolated diarrhoea an allergic reaction, the negative predictive value of the protocol applied in the study was $96 \%$. After $3-5$ years 53 patients (85\%) were contacted and 29 tolerated antibiotic typed in the clinic. No new reactions were reported. As a consequence NPV of the protocol was $97 \%$. 


\section{Table 2. Patients' answers on the telephone follow-up}

\begin{tabular}{|c|c|c|}
\hline \multirow[t]{2}{*}{ Patients' answers on follow-up call } & \multicolumn{2}{|c|}{ Number of patients (\%) } \\
\hline & $\begin{array}{c}\text { 6-12 months after } \\
\text { work-up }\end{array}$ & $\begin{array}{c}3-5 \text { years after } \\
\text { work-up }\end{array}$ \\
\hline Antibiotic typed in the clinic was administered and no reaction occurred & $25(50 \%)$ & $29(55 \%)$ \\
\hline Antibiotic typed in the clinic was administered and reaction occurred & $3(6 \%)$ & $3(6 \%)$ \\
\hline Antibiotic was administered but patient does not know its name; no reaction occurred & $3(6 \%)$ & $4(7 \%)$ \\
\hline Antibiotic was administered but patient does not know its name; reaction occurred & $0(0 \%)$ & $0(0 \%)$ \\
\hline No antibiotics were administered because there were no indications for them & $15(30 \%)$ & $12(23 \%)$ \\
\hline No antibiotics were administered because I was still afraid of them & $3(6 \%)$ & $4(7 \%)$ \\
\hline $\begin{array}{l}\text { No antibiotics were administered because my doctor tried to avoid them in spite of typing a } \\
\text { safe antibiotic }\end{array}$ & $1(2 \%)$ & $1(2 \%)$ \\
\hline
\end{tabular}

\section{Discussion}

IgE-mediated reactions to antibiotics are usually characterized by urticaria, angioedema, itching, shortness of breath, wheezing and a drop in blood pressure. Symptoms develop immediately, most often within 1 hour of drug intake, and may be life-threatening. However the vast majority of adverse reactions to antibiotics are non-immunologic and their clinical manifestation is usually mild. Nevertheless, they often raise confusion among doctors and make patients anxious. In many cases the term "allergy" is applied in an uncritical fashion to all the possible reactions and, as a consequence, penicillins and cephalosporins are unnecessarily avoided. Thus, it is necessary to find safe and reliable protocols of finding antibiotics for future therapy in the extremely heterogenous group of patients with histories of wide spectrum of reactions - from severe anaphylaxis to mild, non-allergic adverse reactions.

The typing of a drug for use in an undefined future was quite controversial. In the 1980s, it was believed that the results of testing should not be used for more than 72 hours because even the patients with negative skin tests could be sensitized after environmental exposure, and the course of their allergy was unpredictable [9]. However, in the following years it was demonstrated that sensitization to penicillins is not common. In the study of Lopez-Serrano and co-workers, 207 patients with negative penicillin skin tests and challenges had a repeated work-up after 10-30 days and conversion to positive skin tests was observed only in 5 subjects [10]. Solensky included into his study 46 patients with a positive history of allergy and negative skin tests. They were given a 10-day course of oral penicillin with repeated skin tests that were all negative [11]. Finally, in the study of Macy et al., 568 patients with a history of an adverse reaction and negative skin tests with penicillin were followed-up over about 4 years and $88.6 \%$ did not react to oral penicillin during the observation [12].

We included into this study a varied group of patients who were referred to our clinic with the suspicion of an allergy to amoxicillin which is now the most commonly used beta-lactam in Poland. In the traditional approach the emphasis is usually put on confirming the suspicion of allergy with the use of skin tests, in vitro tests and provocations [6]. As a consequence, patients are provided with information on "forbidden" antibiotics but they are still very anxious about using an alternative drug. Thus, we aimed at assessing a protocol for typing an antibiotic for a future therapy. We divided our subjects into three groups on the basis of the history of the reaction. Amoxicillin was tested in group A (patients with a low risk of true allergy) - where vast majority showed negative results. The work-up ensured them that the reported symptoms had not resulted from a drug allergy and amoxicillin can be administered safely if necessary. In patients with a history of urticaria/angioedema (group B), cefuroxime was tested. We decided to use cefuroxime because it may be used in numerous clinical situations and is available in both oral and intravenous forms. Cross-reactivity between penicillins and cephalosporins has been shown and often results from similarities in the structure of side chains from similarities in the structure of side chains [13]. However, cross-reactions are now observed less often than previously described which may be partly explained by the fact that early cephalosporin antibiotics contained traces of penicillin $[14,15]$. In the study of Macy et al. in penicillin 
skin test positive individuals, the adverse drug reaction rate was similar with cephalosporin and non-beta-lactam use [16]. In our study, 18 out of 22 patients from group B tolerated cefuroxime. Finally, 17 patients with amoxicillin-induced anaphylaxis were provoked with macrolide and 14 had no reaction.

In the present study we evaluated the NPV of the protocol based on SPT, ICT and DPT. When typing beta-lactams (amoxicillin and cefuroxime) we started with SPT and ICT. In cases with negative results we proceeded to DPT, as described by other authors [17]. In protocols used in Western Europe skin tests with penicylloil polylysine (PPL) and minor determinants mixture (MDM) are routinely performed at the same time [6]. However, in Poland major and minor penicillin determinants are not available, so we were not able to follow this protocol. Also, we have not determined sIgE against penicillins and cephalosporins, which resulted from the low sensitivity of this test [18]. In case of macrolides, skin tests are generally considered unreliable, so the typing included provocation testing only $[19,20]$.

The protocol applied in this study was very safe as we had no anaphylactic reactions, and the positive responses during DPTs were mostly limited to skin reactions. We found a low number of positive results in both skin tests and DPTs probably due to the fact that the culprit drug was tested only in group A, with the history of not being so indicative of true allergy. The comparison of sensitivity and specificity of skin tests and drug challenges was not possible as for provocations we only had selected substances that did not yield reactions in SPT and ICT.

The follow-up telephone visits were successful in 53 (85\%) participants. Among the patients we contacted, 4 tried to avoid the therapy with an antibiotic even despite the clear indication for using it and 1 was not prescribed antibiotic by a family doctor who claimed that there is still some risk in taking it. That might reflect the high level of stress and anxiety resulting from the history of anaphylaxis. 32 participants were given a typed antibiotic, most of them because of respiratory tract infections. In the vast majority of cases no allergic reactions were observed. Our result is in line with the multicentre study of P. Demoly et al. [6]. It included 456 patients who were challenged with several beta-lactams, depending on the history of hypersensitivity. In the follow-up, 109 out of 118 participants reported no allergic reaction to the tested antibiotic, so the NPV was 94\%. Although this protocol differed from the one used in our study, both of them showed a high value of stepwise typing of a safe antibiotic.

Our study has assessed the typing of antibiotics in 'real life' settings where patients' safety is the priority. Nevertheless, it has some limitations. The most important one is the fact that we have not confirmed allergy to amoxicillin in patients with a history of amoxicillin-induced anaphylaxis. They were left with the suspicion of an allergy for the rest of their lives. On the other hand severe anaphylaxis is regarded as contraindication for provocation tests in most guidelines, including ENDA recommendations [8]. Another limitation is the lack of tests with clavulate. Although isolated hypersensitivity to clavulate has been described and a part of our group had a history of a reaction to amoxicillin with clavulate, it is not available in the form of a solution for skin testing and provocations [21].

We also shared problems that are common in most of the studies on the allergy to antibiotics. They are a rather rarely used group of drugs, so in the follow-up many patients cannot assess their safety. In our study, $23 \%$ of participants did not use any antibiotics during the follow-up period because they were not necessary. That reduced the number of answers on tolerability of the drug typed, but our proportion is still low compared to other studies [6]. The question of how long the results are predictive is still current, but the majority of patients seem to tolerate the tested antibiotics for a long period of time. Finally, what makes diagnosing of drug allergy very complicated is the influence of cofactors such as viral infection, physical exercise or alcohol. Their role was not assessed in our group.

\section{Conclusion}

The stepwise approach including skin prick tests, intracutaneous tests and provocations with amoxicillin, cefuroxime and clarithromycin is a safe procedure and allows typing an antibiotic for the use in real life clinical situations in a vast majority of patients.

\section{Conflict of interest}

The authors declare no conflict of interest.

\section{References:}

1. Hryniewicz W, Ozorowski T, Radzikowski A. Rekomendacje posteppowania $\mathrm{w}$ pozaszpialnych zakażeniach układu oddechowego. www.antybiotyki.edu.pl/pdf/RekomendacjeA42009. pdf; 10.05 .2010 .

2. Lim W, Baudouin S, George R et al. BTS guidelines for the management of community aquired pneumonia in adults: up- 
date 2009. Thorax 2009; 64 (Suppl 3): 1-55. doi: 10.1136/ thx.2009.121434

3. Lutomski D, LaFollette J, Biaglow $\mathrm{M}$ et al. Antibiotic allergies in the medical record: effect on drug selection and assessment of validity. Pharmacotherapy 2008; 28: 1348-1353. doi: 10.1592/phco.28.11.1348.

4. Bigby M, Jick S, Jick H et al. Drug induced cutaneous reactions. JAMA 1986; 256: 3358-3363.

5. Blanca M, Romano A, Torres M et al. Update on evaluation of hypersensitivity reactions to betalactams. Allergy 2009; 64: 183-193. doi: 10.1111/j.1398-9995.2008.01916.x.

6. Demoly P, Romano A, Botelho C et al. Determining the negative predictive value of provocation tests with beta-lactams. Allergy 2010; 65: 327-332. doi: 10.1111/j.1398-9995.2009.02228.x.

7. Sampson H, Munoz-Furlong A, Campbell R et al. Second symposium on the definition and management of anaphylaxis : summary report: Second National Institute of Allergy and Infectious Disease/Food Allergy and Anaphylaxis Network Symposium. J Allergy Clin Immunol 2006; 117: 391-397.

8. Aberer W, Bircher A, Romano A et al. Drug provocation testing in the diagnosis of drug hypersensitivity reactions : general considerations. Allergy 2003; 58: 854-863.

9. Saxon A, Beall GN, Rohr AS, Adelman DC. Immediate hypersensitivity reactions to beta-lactam antibiotics. Ann Intern Med 1987; 107: 204-215.

10. Lopez-Serrano M, Caballero M, Barranco P et al. Booster responses in the study of allergy reactions to beta-lactam antibiotic. J Invest Allergol Clin Immunol 1996; 6: 30-35.

11. Solensky R, Earl H, Gruchalla R. Lack of penicillin resensitization in patients with a history of penicillin allergy after receiving repeated penicillin courses. Arch Intern Med 2002; 162: $822-826$.
12. Macy E, Mangat R, Burchette R. Penicillin skin testing in advance of need: multiyear follow-up in 568 test result-negative subjects exposed to oral penicillins. J Allergy Clin Immunol 2003; 111: 1111-1115.

13. Romano A, Gueant-Rodriguez RM, Marinella V et al. Cross-reactivity and tolerability of cephalosporins in patients with immediate hypersensitivity to penicillin. Ann Intern Med 2004; 141: 16-22.

14. Blanka M, Romano A, Torres M et al. Update on the evaluation of hypersensitivity reactions to beta-lactams. Allergy 2009; 64: 183-193. doi: 10.1111/j.1398-9995.2008.01916.x.

15. Sastre J, Quijano LD, Novalbos A et al. Clinical cross-reactivity between amoxicillin and cephadroxil in patients allergic to amoxicillin and with good tolerance of penicillin. Allergy 1996; 51: 383-386.

16. Macy E, Burchette R. Oral antibiotic adverse reactions after penicillin skin testing: multi-year follow-up. Allergy 2002; 1151-1158.

17. Bousquet PJ, Pipet A, Bousquet-Rouanet P et al. Oral challenges are needed in the diagnosis of beta-lactam hypersensitivity. Clin Exp Allergy 2007; 38: 185-190.

18. Fontaine C, Mayorga C, Bousquet $\mathrm{P}$ et al. Relevance of the determination of serum-specific IgE antibodies in the diagnosis of immediate beta-lactam allergy. Allergy 2007; 62: 47-52.

19. Seitz CS, Brocker EB, Trautmann A. Suspicion of macrolide allergy after treatment of infectious diseases including Helicobacter pylori: results of allergological testing. Allergol Immunopathol 2011; 39: 193-199. doi: 10.1016/j.aller.2010.06.008.

20. Yoon S, Park S, Kim S et al. Validation of the cephalosporin intradermal skin test for predicting immediate hypersensitivity: a prospective study with drug challenge. Allergy 2013; 68: 938-944. doi: 10.1111/all.12182.

21. Cahen YD, Wuthrich B: Drug allergy to the beta-lactam antibiotics clavulanic acid and amoxicillin. Allergy 1997; 52: 117-118. 